\title{
The Importance of Automated Systems for Information Gathering and Decision Making Support for The Pain Management in Patient with Spinal Cord Injury
}

\section{Nasrolah Nasr Heydarabadi ${ }^{1}$, Reza Safdari2 ${ }^{*}$, Marjan Ghazi Saeedi ${ }^{2}$, Arash Rahman ${ }^{3}$, Laleh Hakemi ${ }^{4}$, Peirhossein Kolivand $^{4}$, Mobin Shaterian ${ }^{5}$}

${ }^{1}$ Department of Medical Informatics, School of Allied Medical Sciences, Tehran University of Medical Sciences, Tehran, Iran ${ }^{2}$ Health Information Management Research Center, Health School of Allied Medical Sciences, Tehran University of Medical Sciences, Tehran, Iran

${ }^{3}$ Department of Computer Engineering, Faculty of Technical Engineering, Roudehen branch, Islamic Azad University, Roudehen. Iran, ${ }^{4}$ Shefa Neuroscience Research Center, Khatam Alanbia Hospital, Tehran, Iran ${ }^{5}$ Department of Computer, Science and Research Branch, Islamic Azad University, Tehran, Iran

\section{A BSTRACT}

Introduction: Pain is a major symptom in many medical conditions and is significantly associated with alteration of the quality of life and individual as well as overall performance. Pain is one of the main problems in patient with spinal cord injury. Spinal cord injury is one of the causes of chronic pain that occurs due to temporary or permanent changes in spinal cord function. Traffic accidents, war injuries and sports injuries are the major reasons. Clinical decision support system separates intelligently expert's information and knowledge for physician, health professionals, and patients as well as other individuals and represents the knowledge in right time to provide more efficient treatment and health care. Automated health information systems are gathering data and information to support the integration of managerial decisionmaking processes. Conclusion: Paper-based systems are not suitable for repetitive clinical evaluations of patients with spinal cord injury. Design and implementation of automated systems for gathering information in pain management and physician assistant to medical decision making for pain diagnosis in patients with spinal cord injury are very effective. This system may use different artificial intelligence techniques, including Bayesian networks, artificial neural network, decision trees, and fuzzy logic.

\section{Key words:}

1. Chronic Pain

2. Spinal Cord Injuries

3. Decision Support

Systems, Clinical

\section{*Corresponding Author: Reza Safdari}

E-mail:Rsafdari@tums.ac.ir 


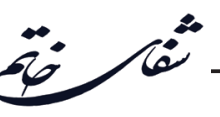

\title{
اهميت سيستمهاى خودكار براى جمع آورى اطلاعات و يشتيبانى تصميمَيرى جهت مديريت درد در بيماران آسيب نخاعى الاعات
}

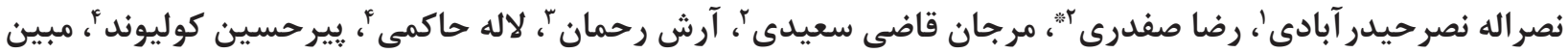

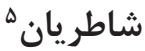 \\ اكروه انفورماتيك بزشكى، دانشكده پِيرايزشكى، دانشكاه علوم يزشكى تهران، تهران، ايران \\ †مركز تحقيقات مديريت اطلاعات سلامت، دانشكده بهداشت بيرايزشكى، دانشكاه علوم يزشكى تهران، تهران، ايران

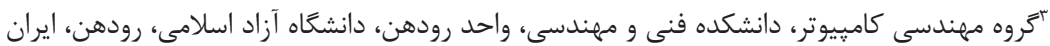 \\ †مركز تحقيقات علوم اعصاب شفا، بيمارستان خاتمالانبياء، تهران، ايران

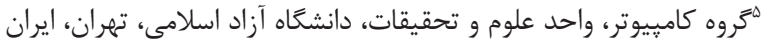

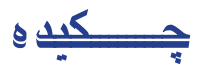

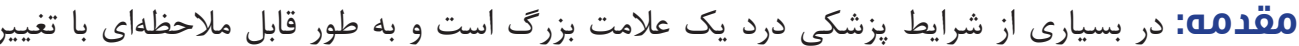

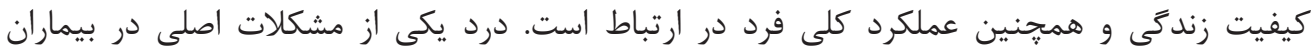

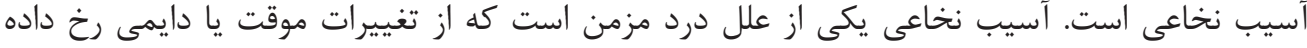

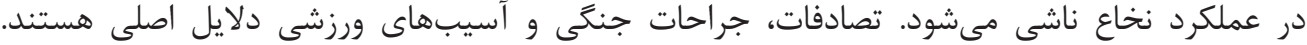

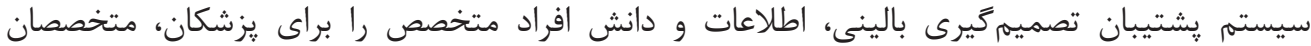

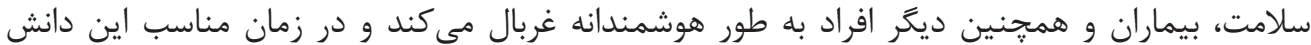

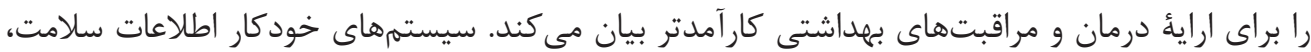

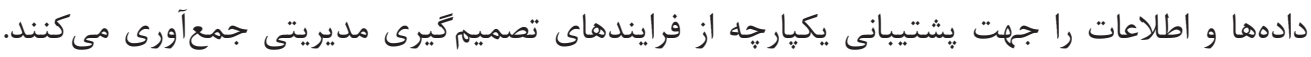

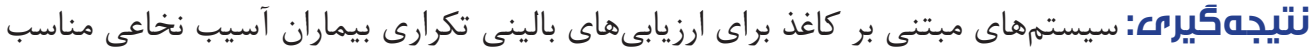

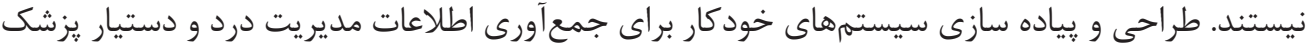

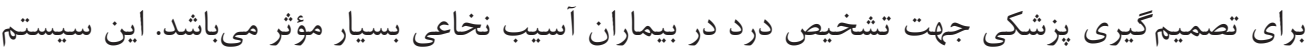

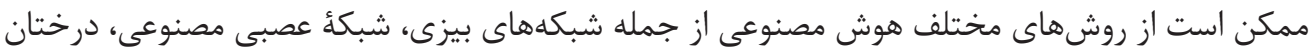
تصميم و منطق فازى استفاده كند.

كليد وازهها:

1

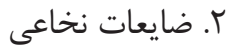
"ا. سيستمهاى يشتيبان تصميمزيـرى بالينى

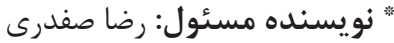
آدرس الكترونيكى: Rsafdari@tums.ac.ir 


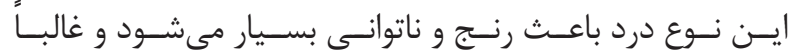

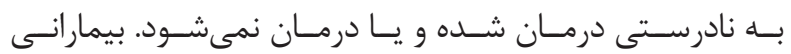

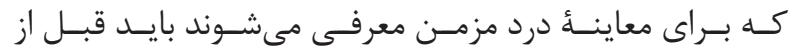

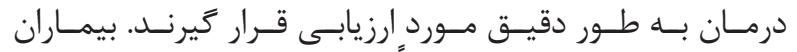

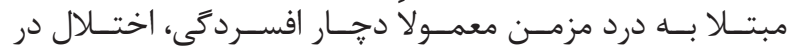

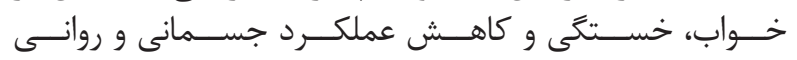

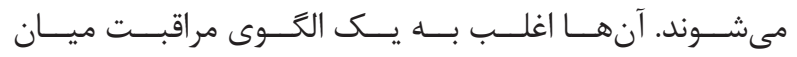

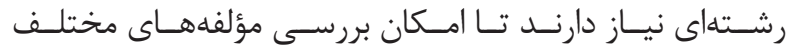

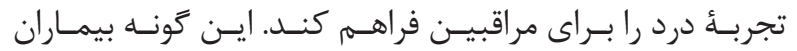

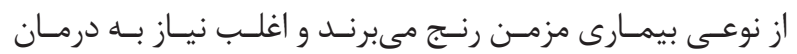

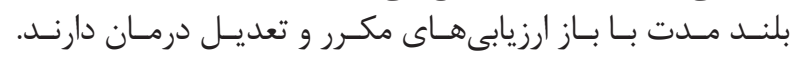

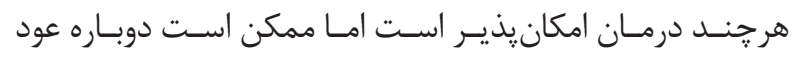

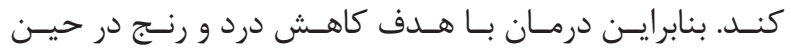

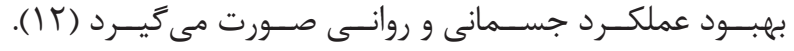

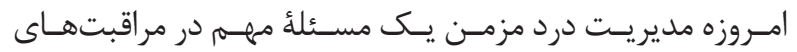

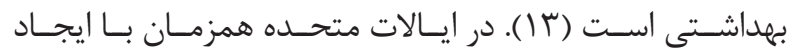

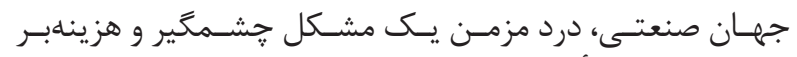

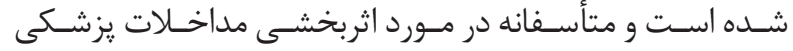

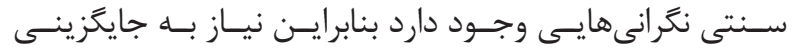

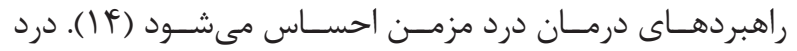

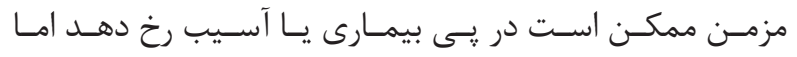

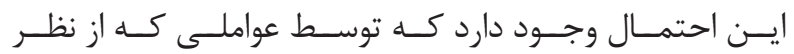

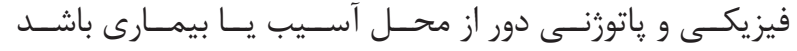

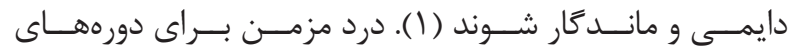

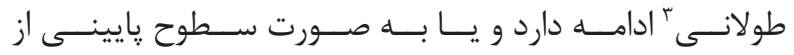

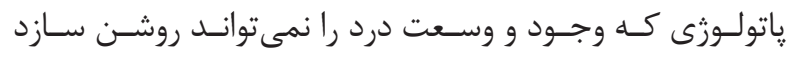

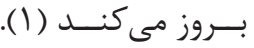

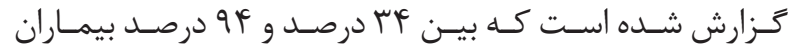

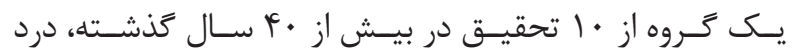

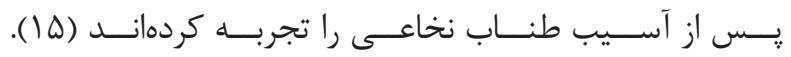

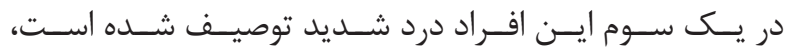

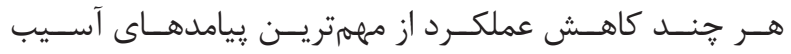

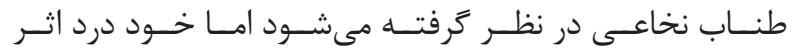

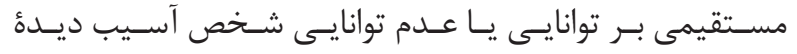

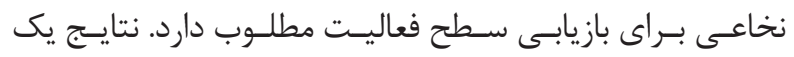

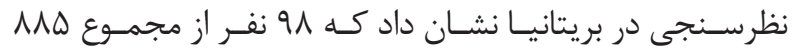

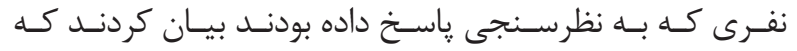

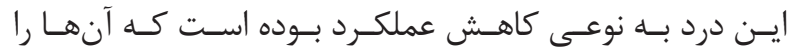

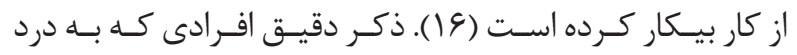

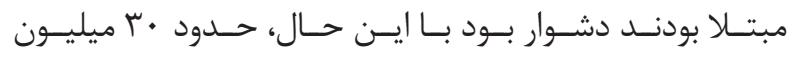

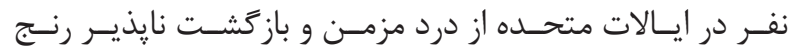

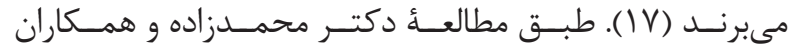

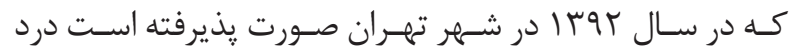

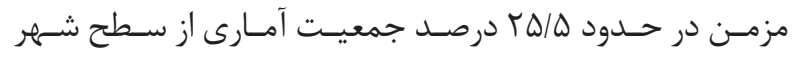

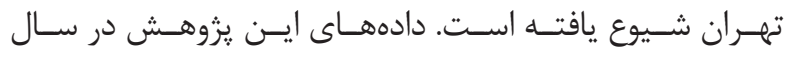

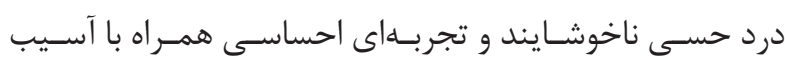

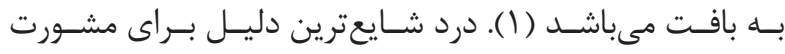

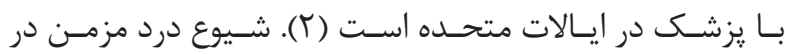

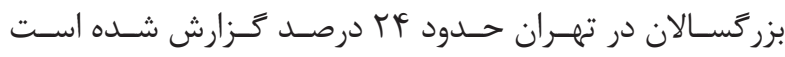

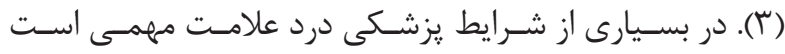

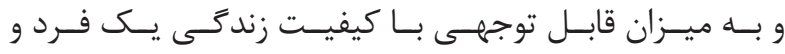

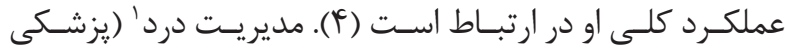

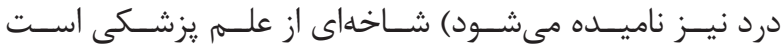

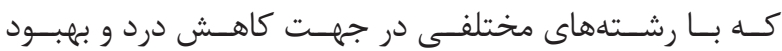

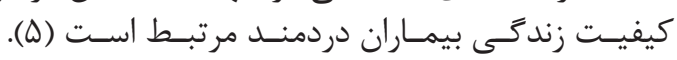

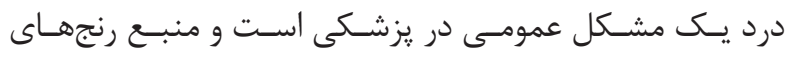

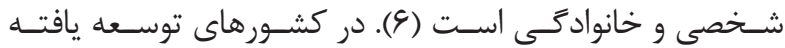

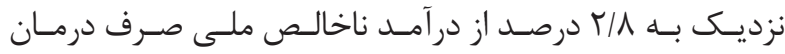

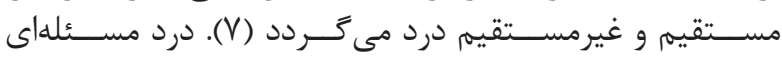

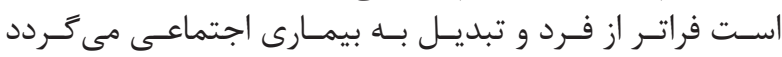

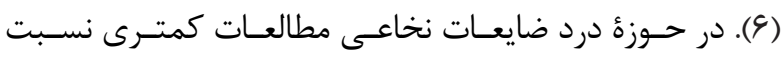

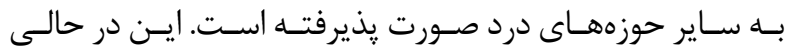

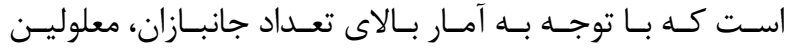

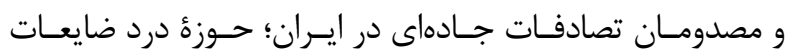

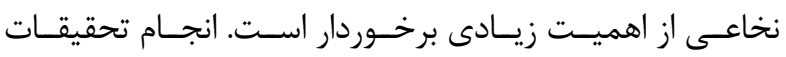

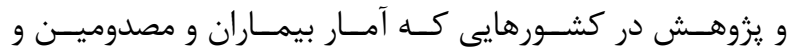

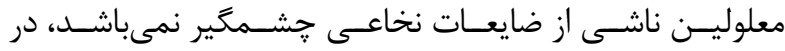

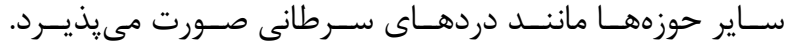

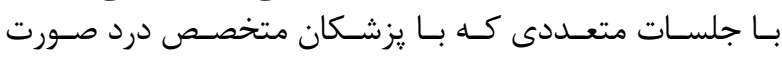

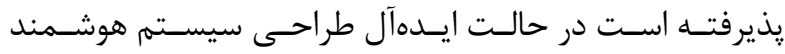

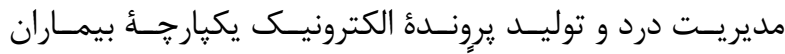

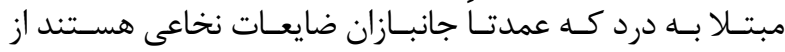

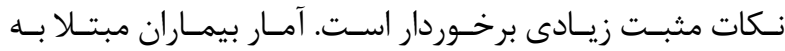

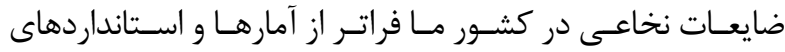

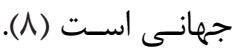

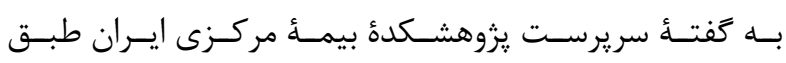

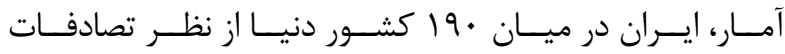

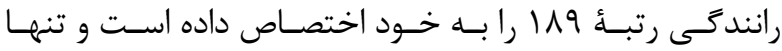

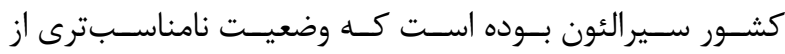

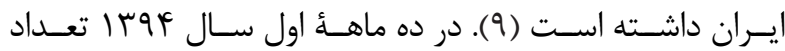

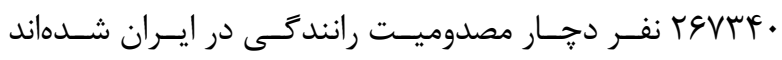

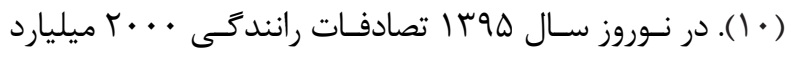

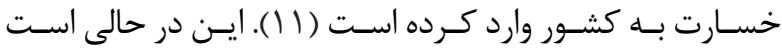

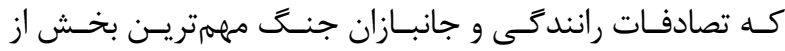

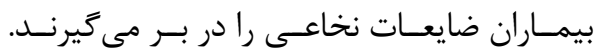

درد مزمن

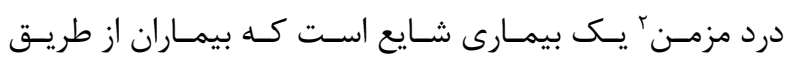

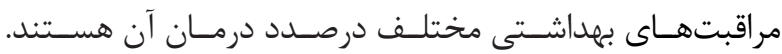

${ }^{1}$ Pain management

${ }^{2}$ Chronic pain

${ }^{3}$ Long term 
تيم مديريت درد

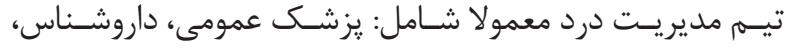

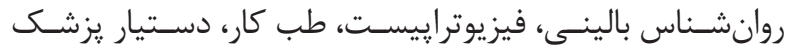

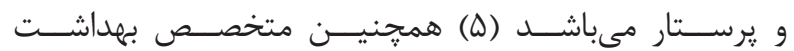

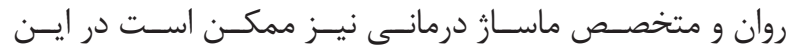

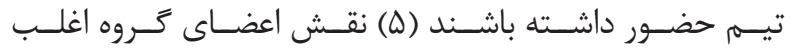

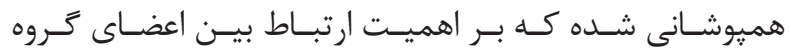

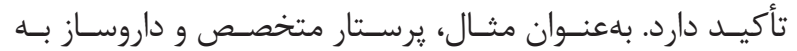

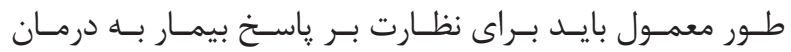

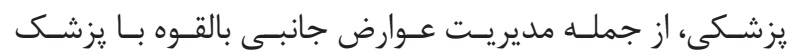

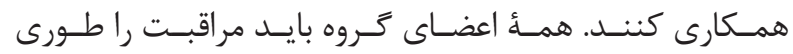

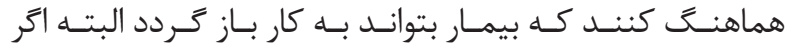

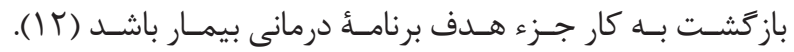

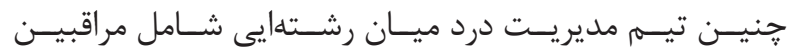

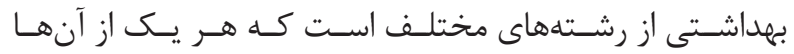

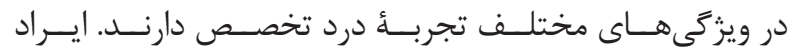

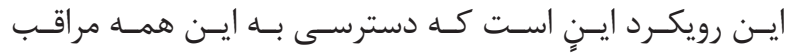

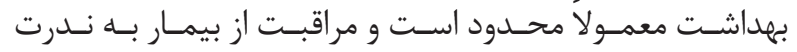

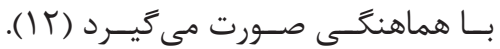

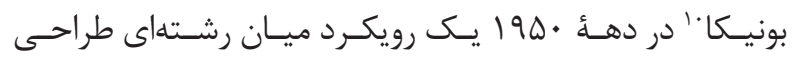

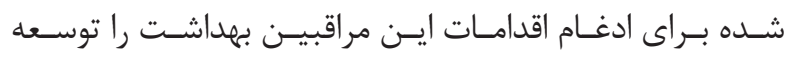

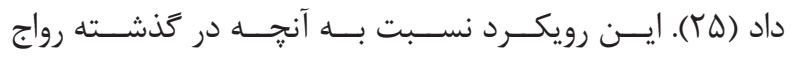

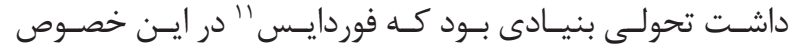

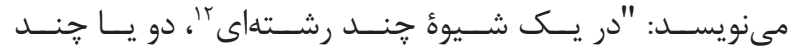

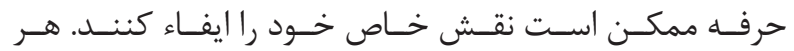

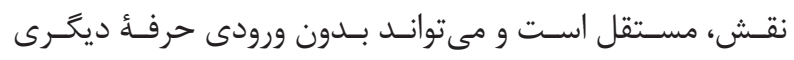

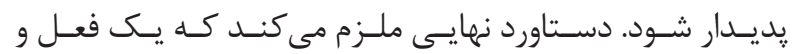

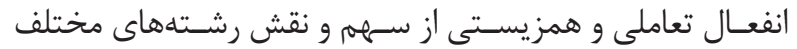

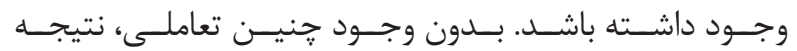

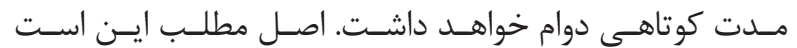

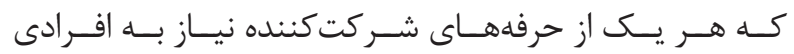

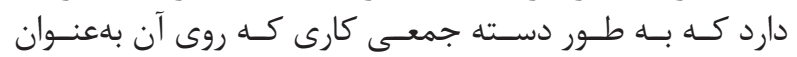

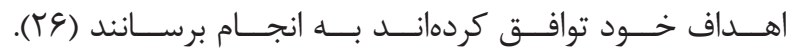

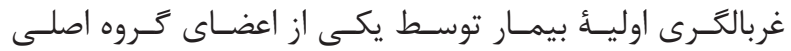

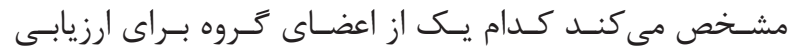

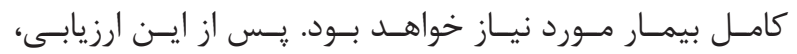

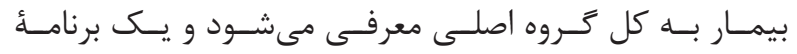

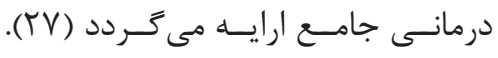

مر اكز "جند تخصصى درد

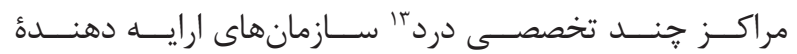

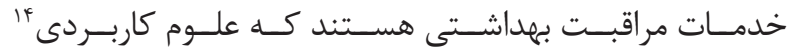

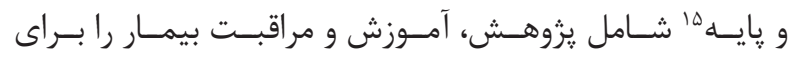

${ }^{4}$ Transverse myelitis

${ }^{5}$ Polio

${ }^{6}$ Spina bifida

${ }^{7}$ Friedreich's ataxia

${ }^{8}$ Paralysis

${ }^{9}$ Spinal cord injury

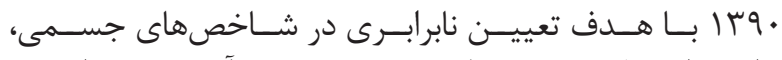

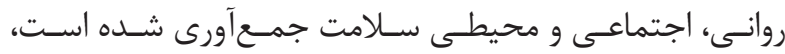

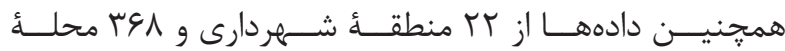

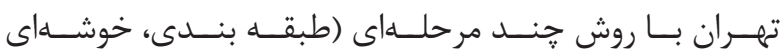

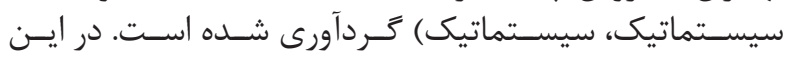

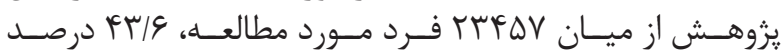

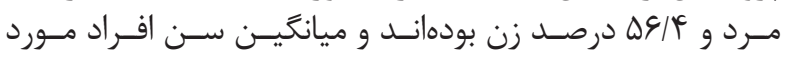

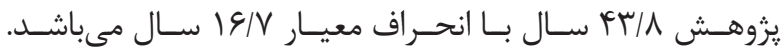

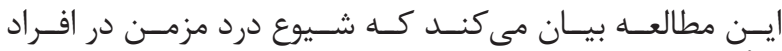

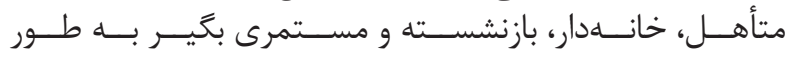

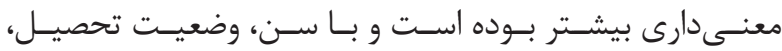

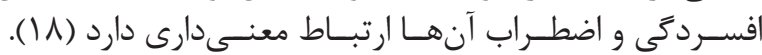

در ميــان دردهــاى رصــ شـده در ايسـن مطالعـه، درد مزمــن

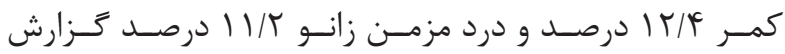

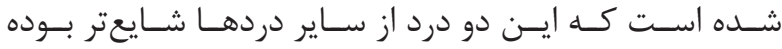

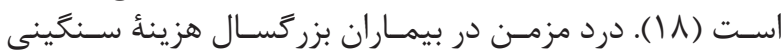

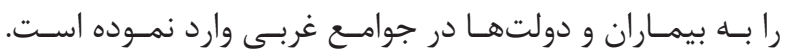

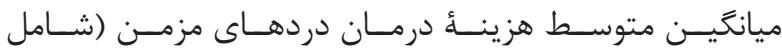

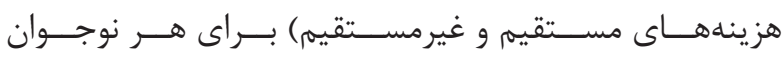

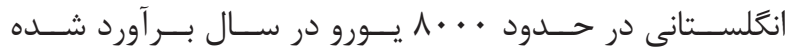

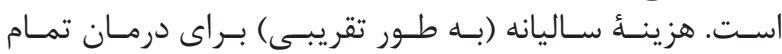

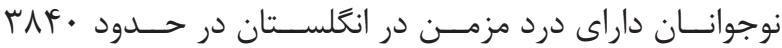

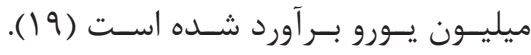

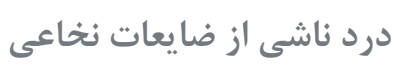

ضايعـات نخاعـى، يـك آســيب بـهـ نخــاع و در نتيجــه تغييـر

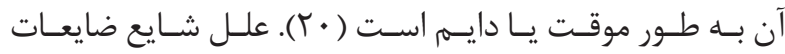

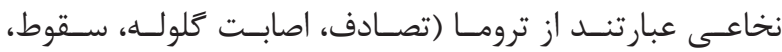

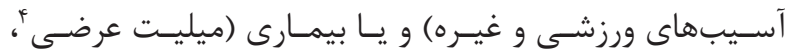

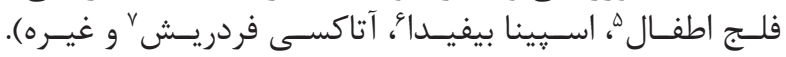

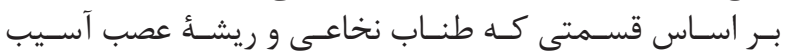

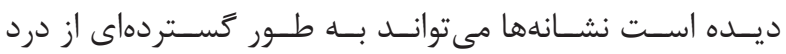

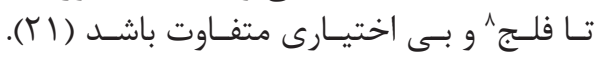

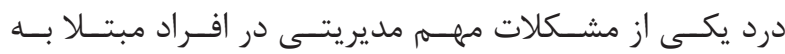

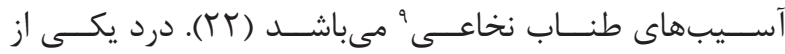

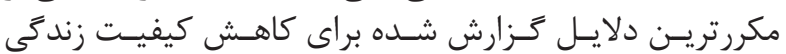

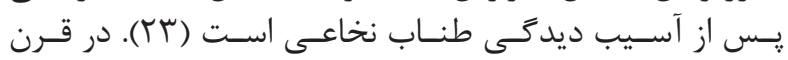

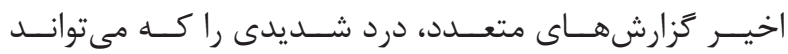

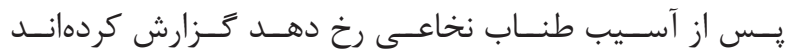

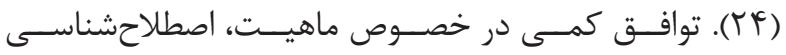

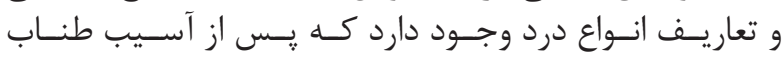

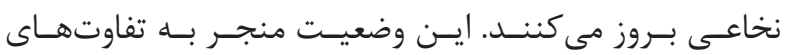

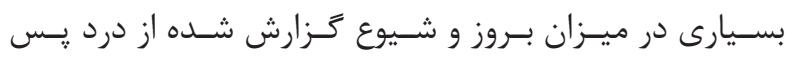

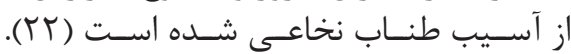

\footnotetext{
${ }^{10}$ Bonica

${ }^{11}$ Fordyce

${ }^{12}$ Multidisciplinary

${ }^{13}$ Multidisciplinary (Interdisciplinary) pain center

${ }^{14}$ Applied

${ }^{15}$ Basic
} 


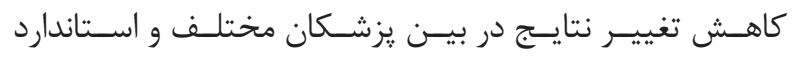

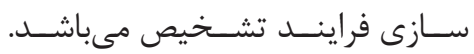

سيستم مديريت درد

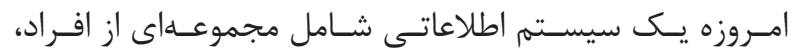

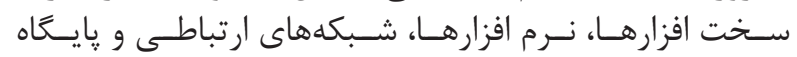

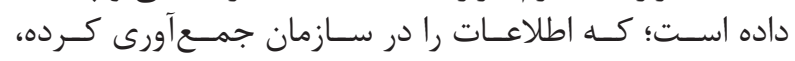

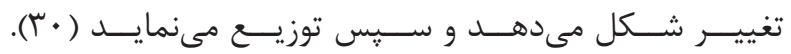

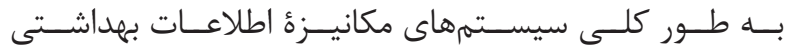

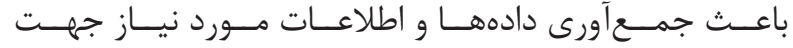

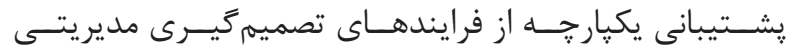

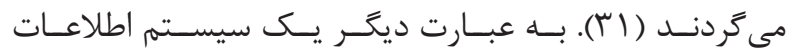

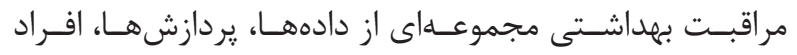

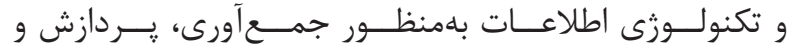

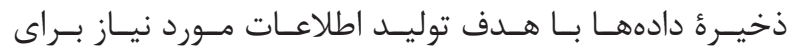

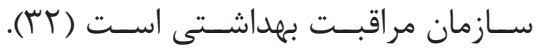

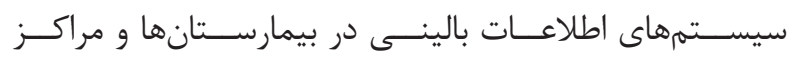

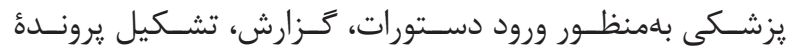

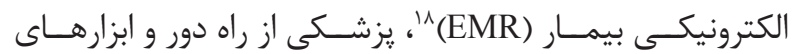

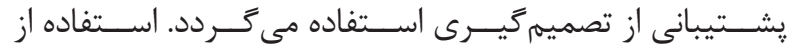

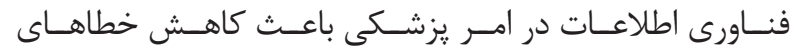

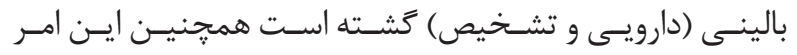

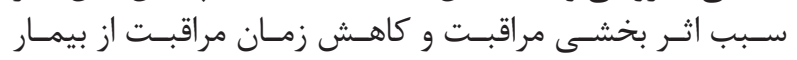

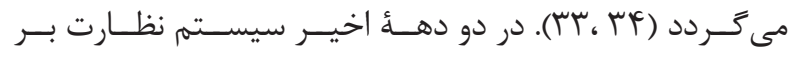

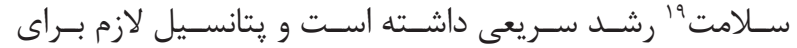

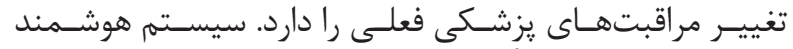

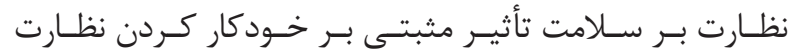

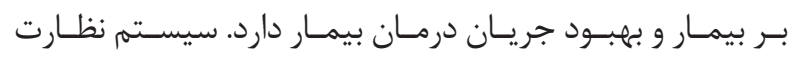

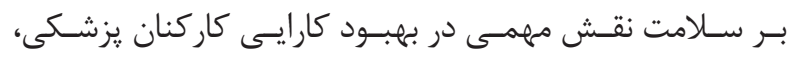

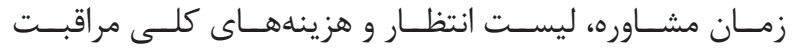

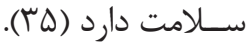

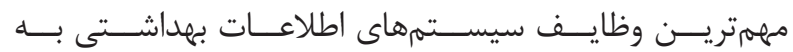

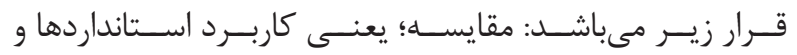

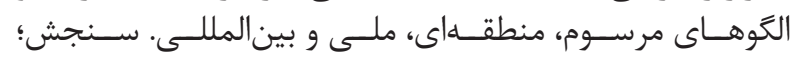

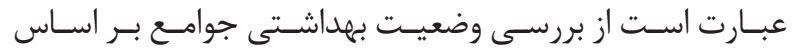

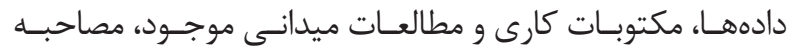

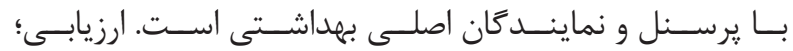

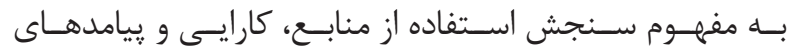

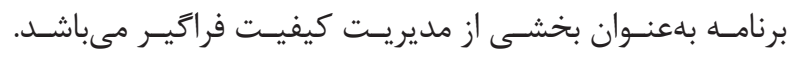

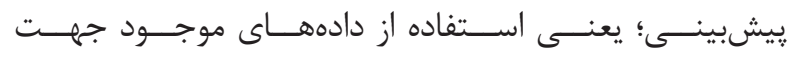

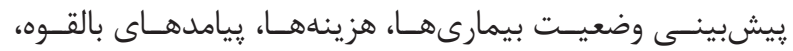

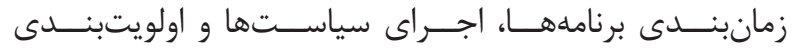

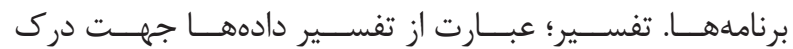

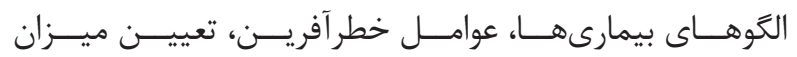

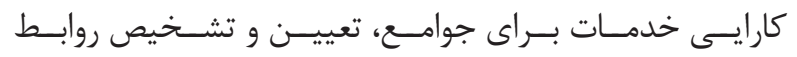

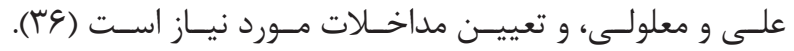

${ }^{16}$ Interventional

${ }^{17}$ Fluoroscopy

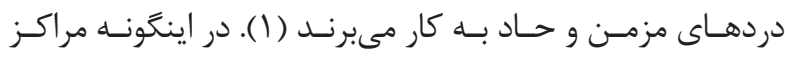

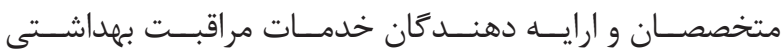

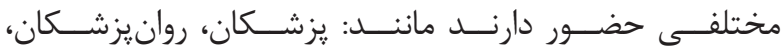

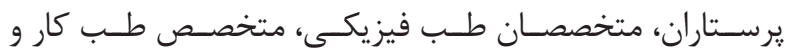

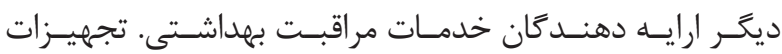

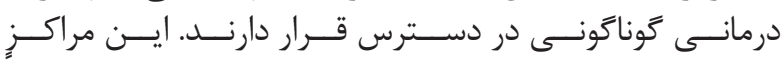

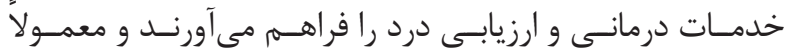

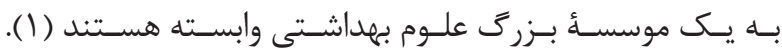

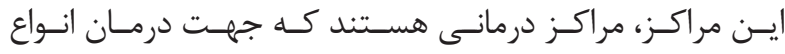

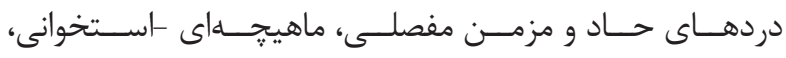

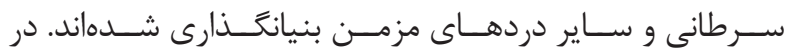

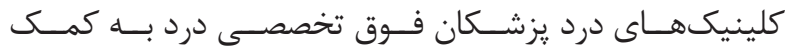

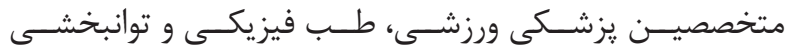

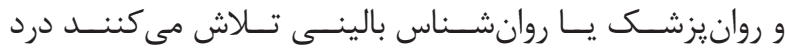

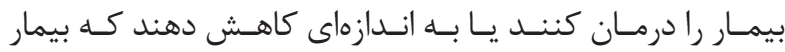

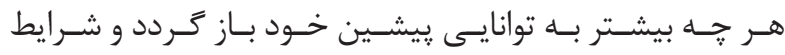

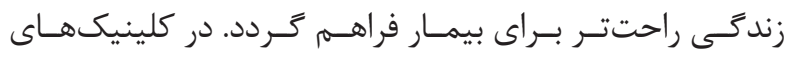

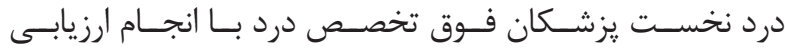

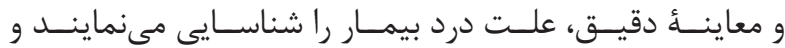

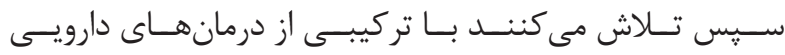

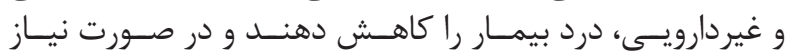

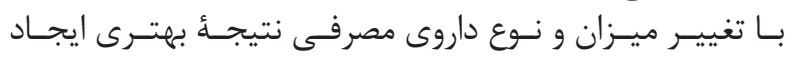

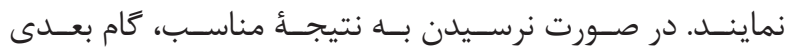

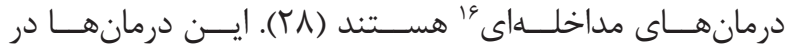

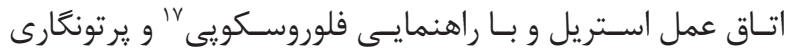

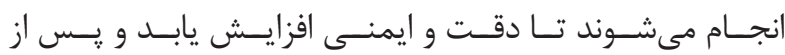

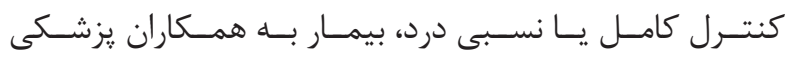

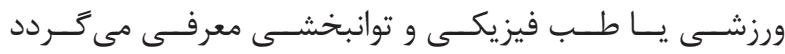

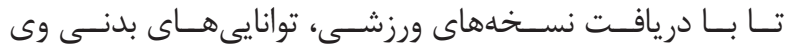

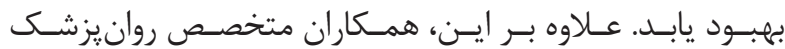

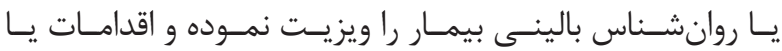

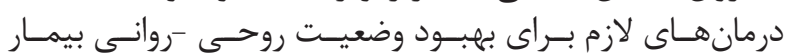

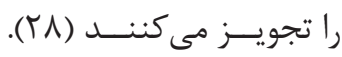

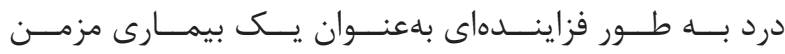

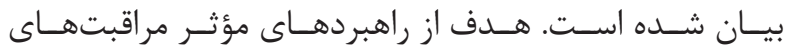

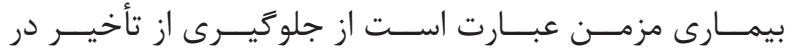

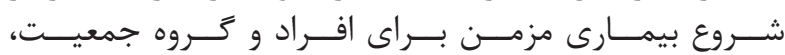

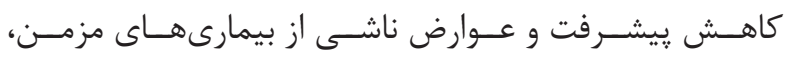

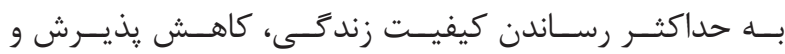

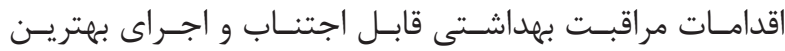

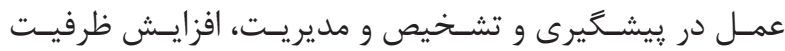

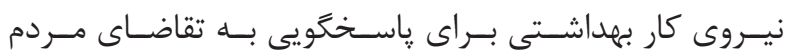

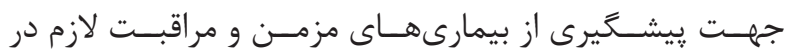

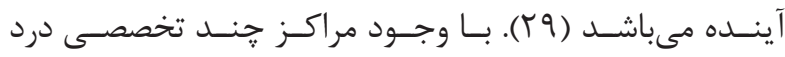

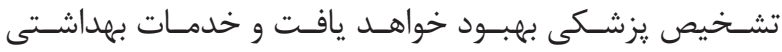

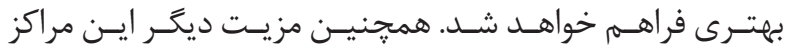

${ }^{18}$ Electronic medical record

${ }^{19}$ Health monitoring systems 


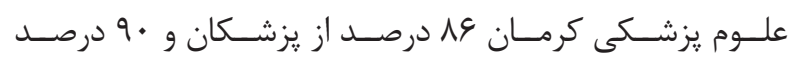

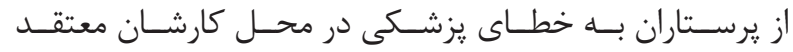

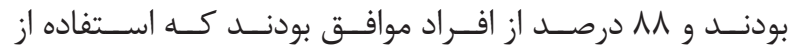

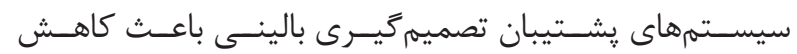

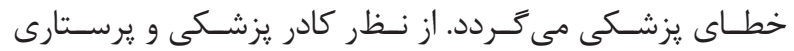

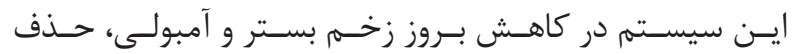

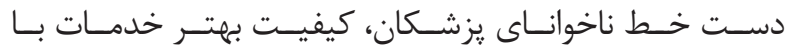

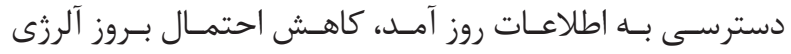

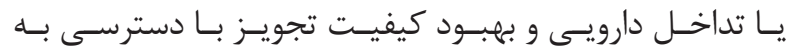

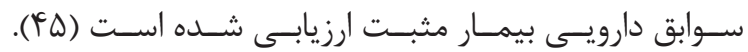

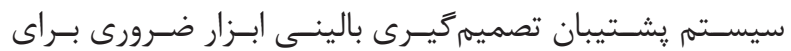

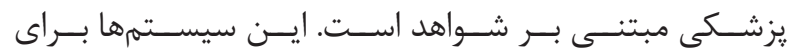

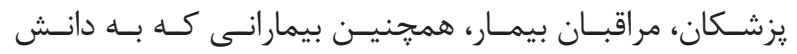

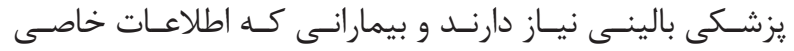

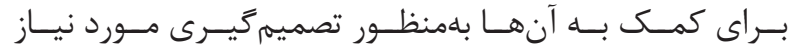

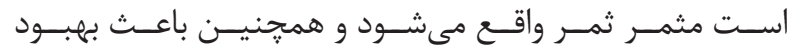

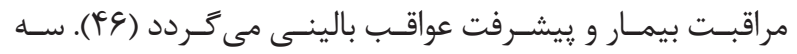

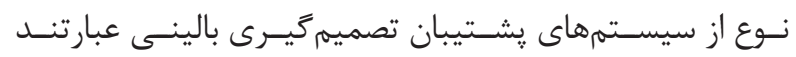

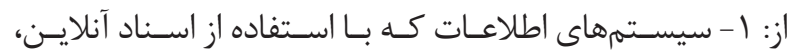

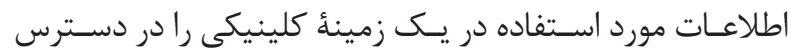

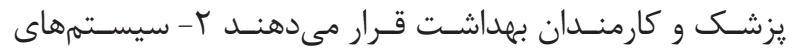

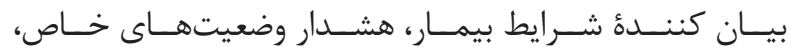

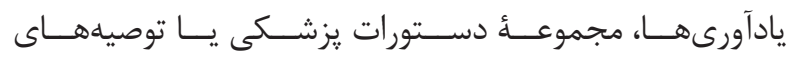

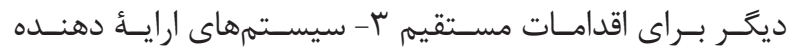

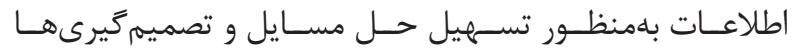

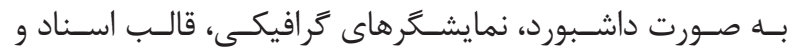
كزارشـات سـاختار يافتـهـ (FT)

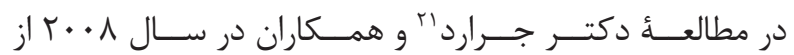

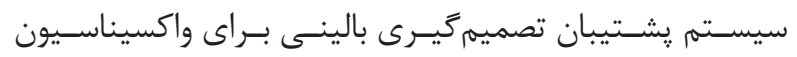

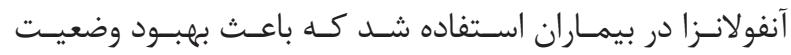

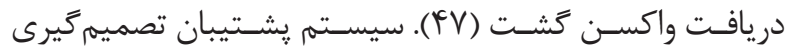

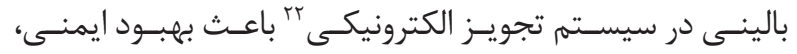

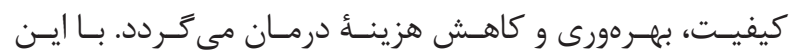

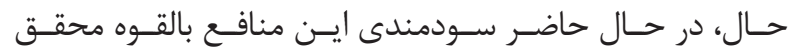

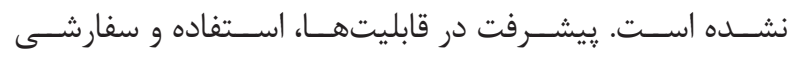

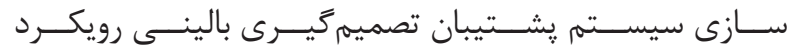

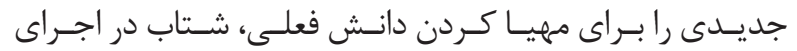

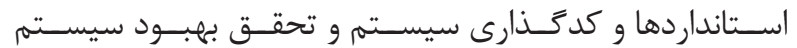

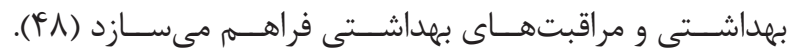

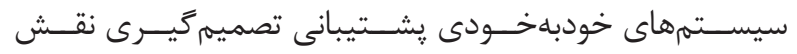

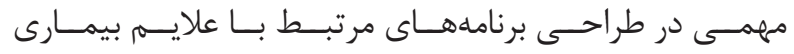

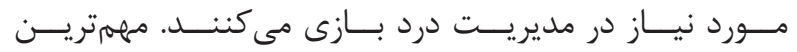

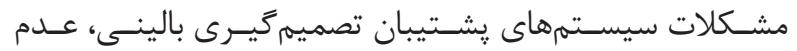

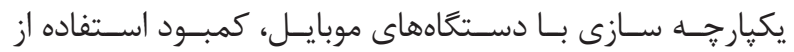

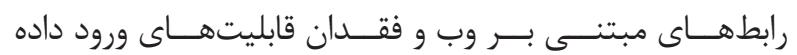

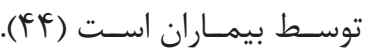

${ }^{20}$ Clinical decision support system

${ }^{21}$ Gerard

${ }^{22}$ Electronic prescribing or e-prescribing (e-Rx)
سيسـتمهاى اطلاعـات فراينــد ارتباطـات را تسـهيل و اطلاعات

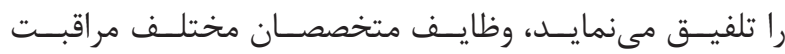

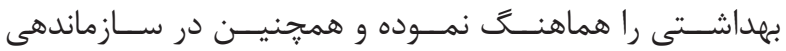

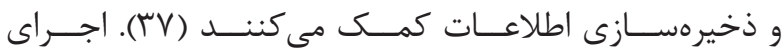

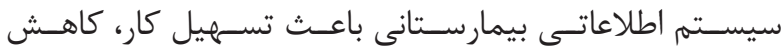

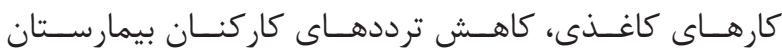

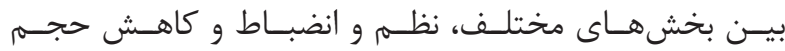

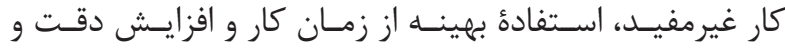

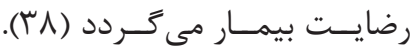

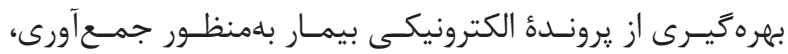

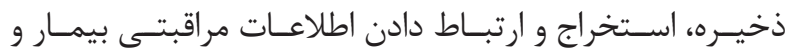

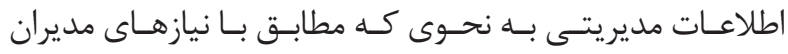

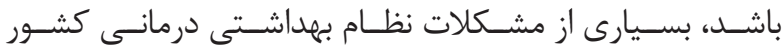

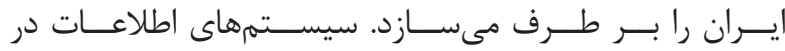

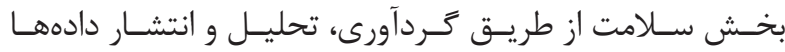

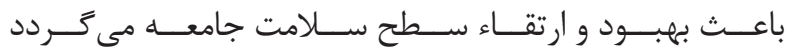

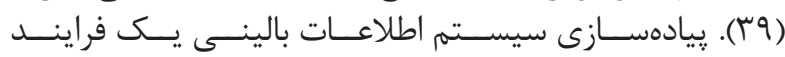

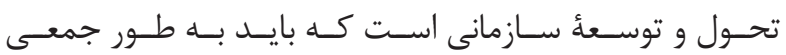

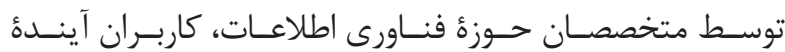

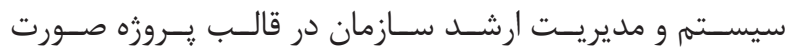

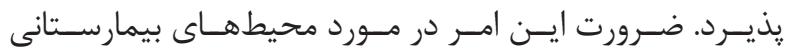

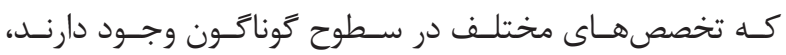

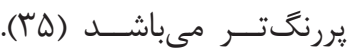
سيستممهاى هشتيبان تصميم گيرى بالينى

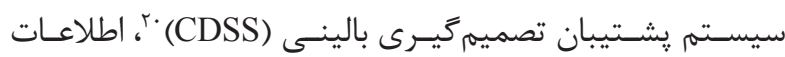

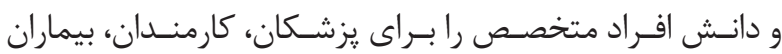

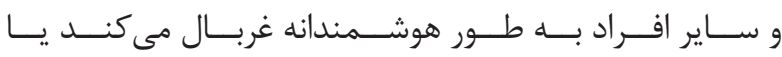

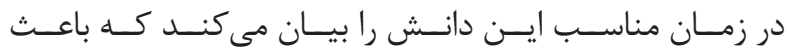

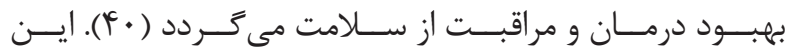

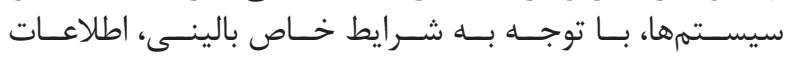

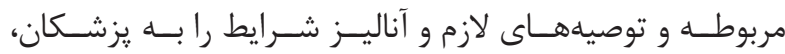

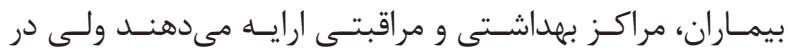

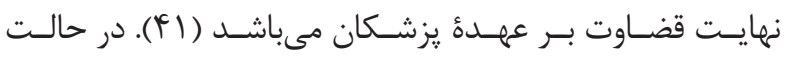

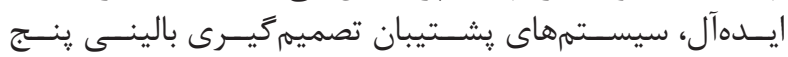

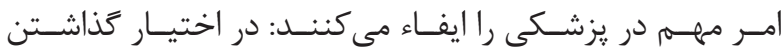

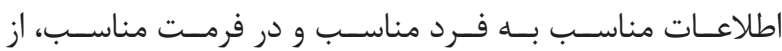

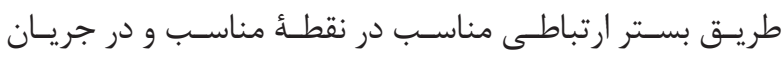

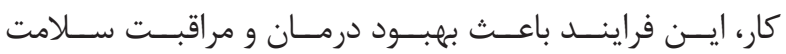

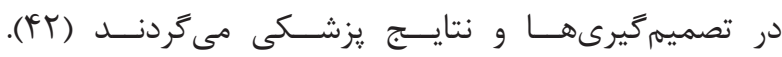

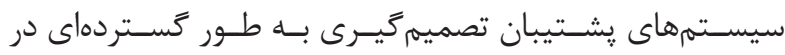

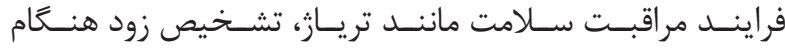

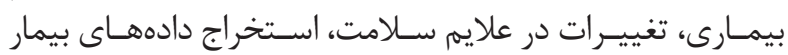

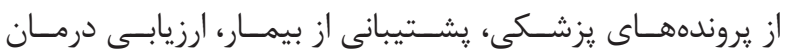

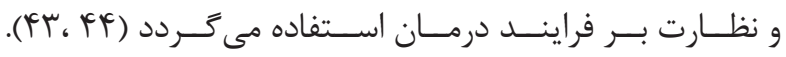
طبــق بررســى انجــام شــده در يـــ يزوهـــ در دانشـــاه 


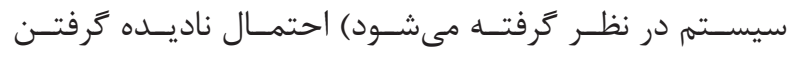

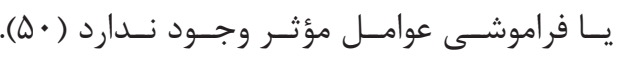

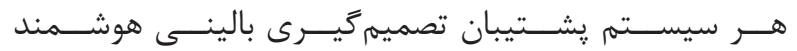

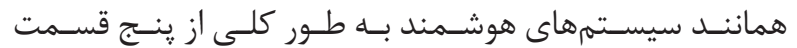

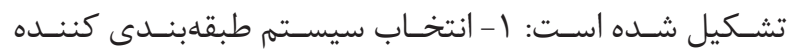

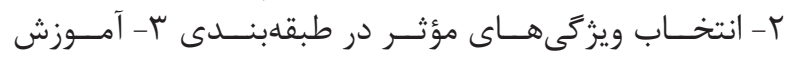

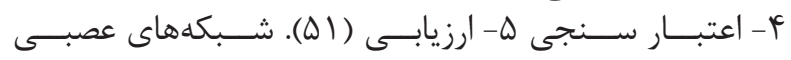

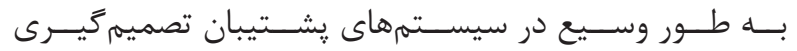

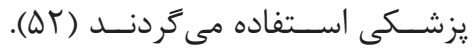

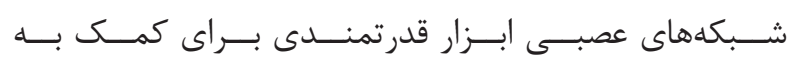

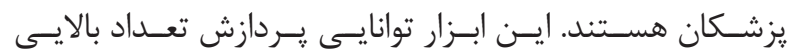

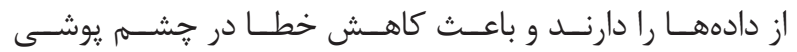

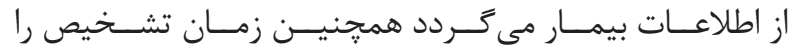

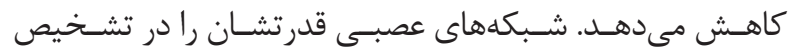

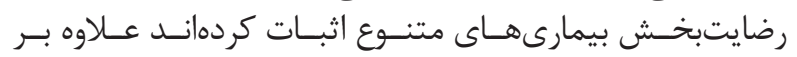

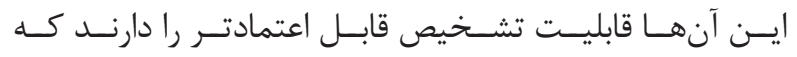

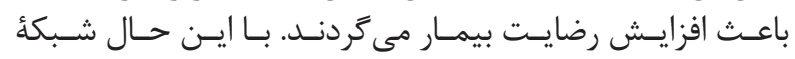

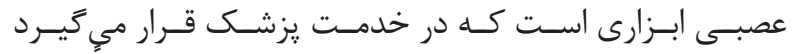

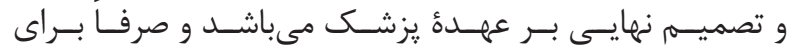

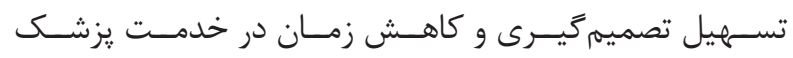

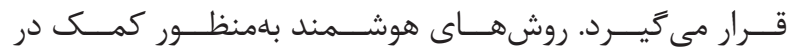

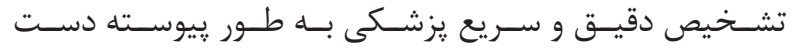

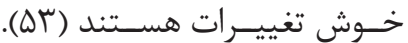

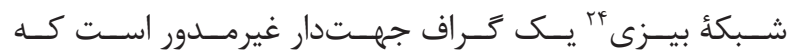

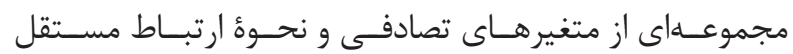

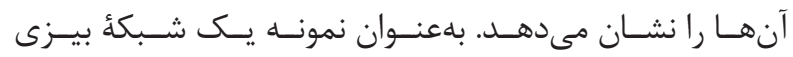

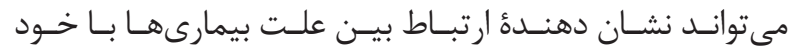

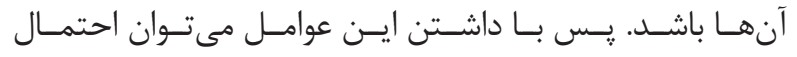

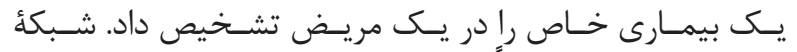

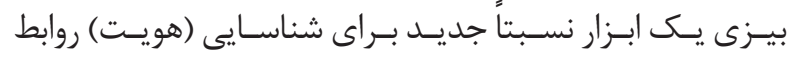

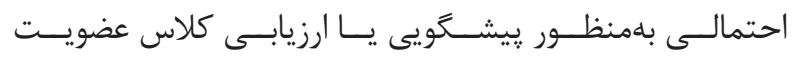

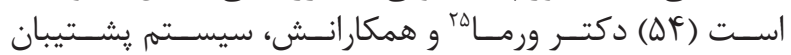

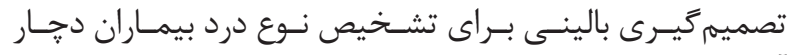

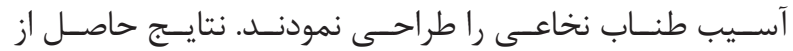

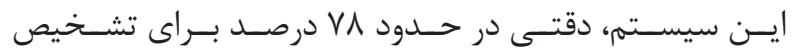

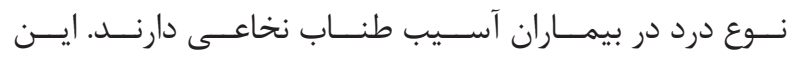

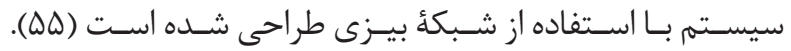

\section{نتيجه كَيرى}

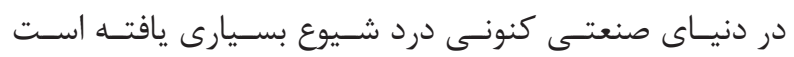

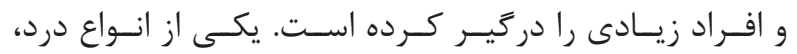

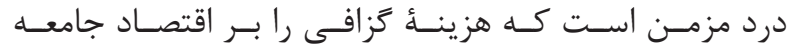

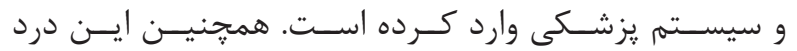

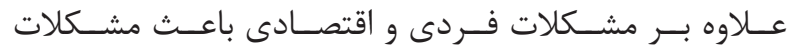

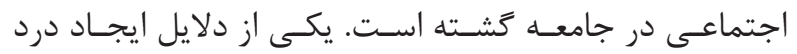

${ }^{23}$ Pombo

${ }^{24}$ Bayesian network

${ }^{25}$ Verma

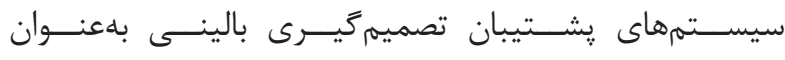

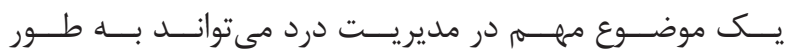

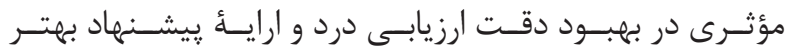

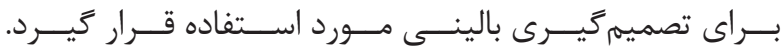

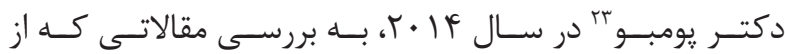

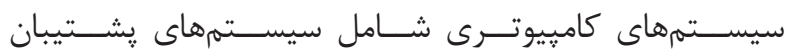

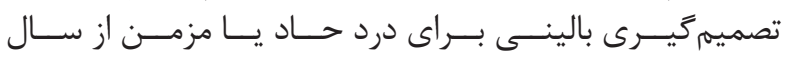

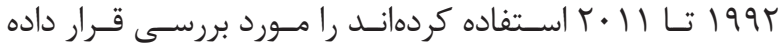

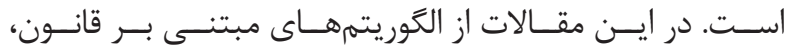

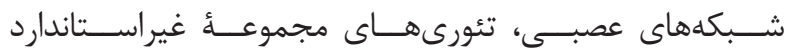

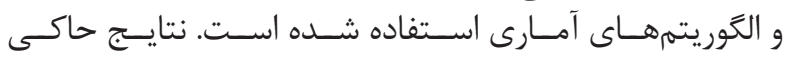

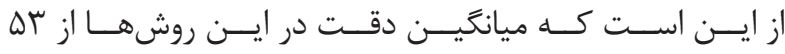

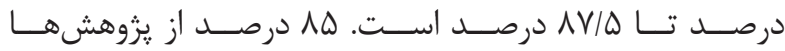

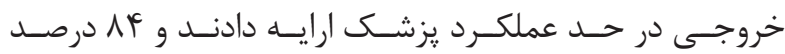

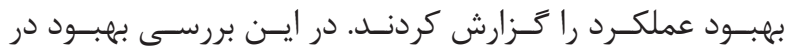

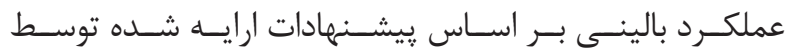

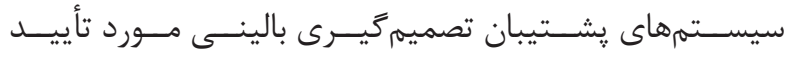

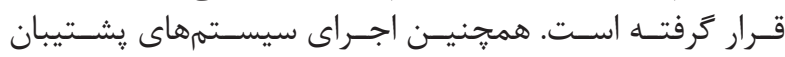

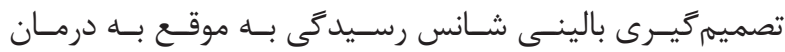

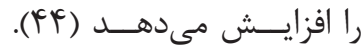

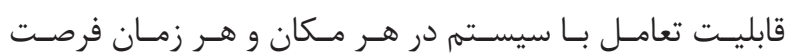

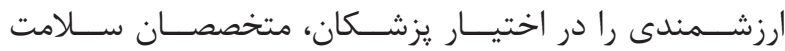

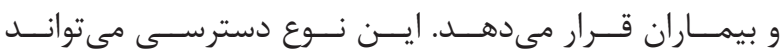

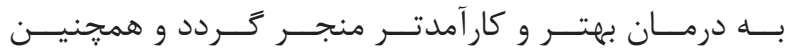

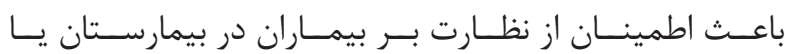

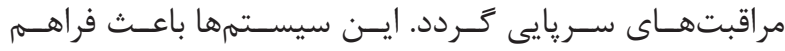

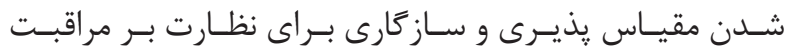

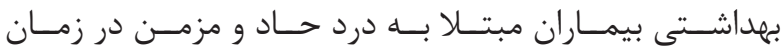

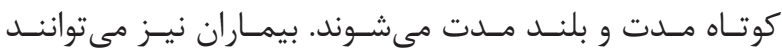

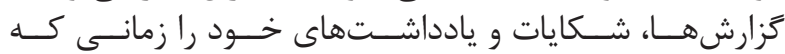

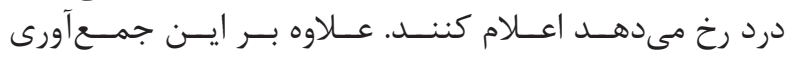

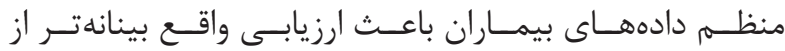

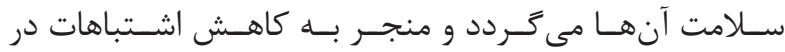

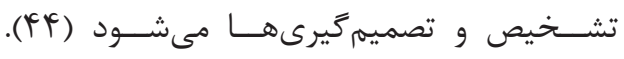

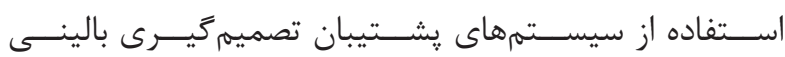

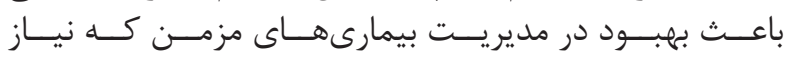

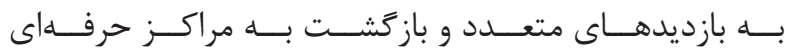

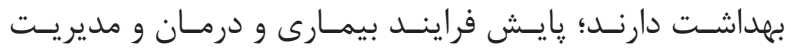

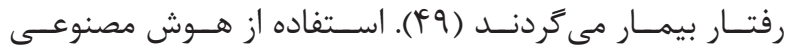

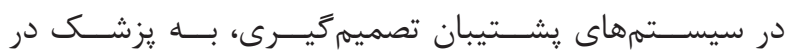

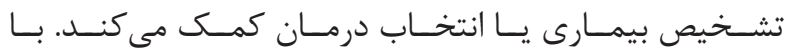

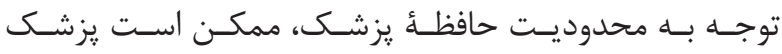

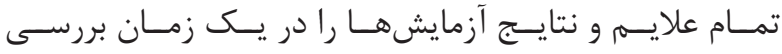

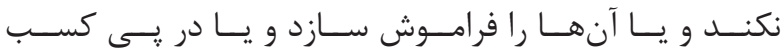

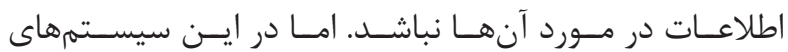

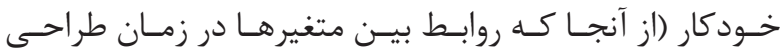




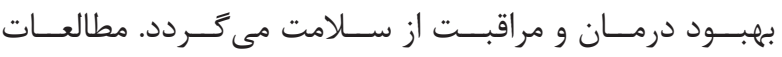

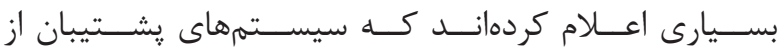

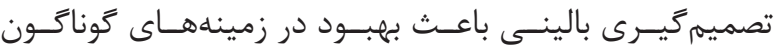

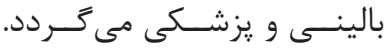

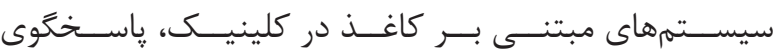

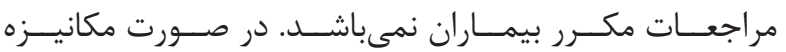

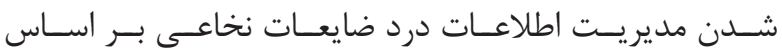

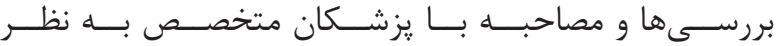

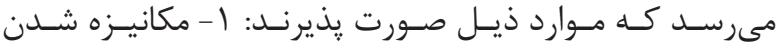

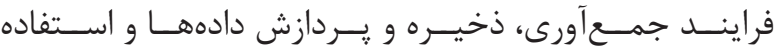

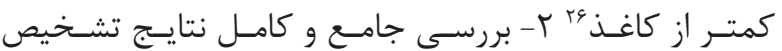

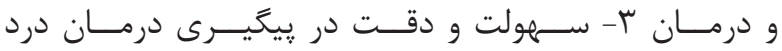

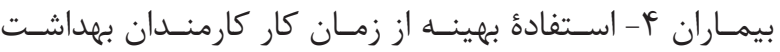

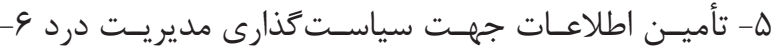

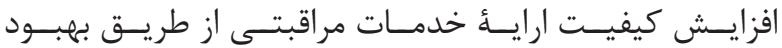

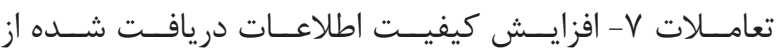

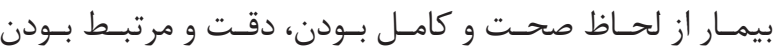

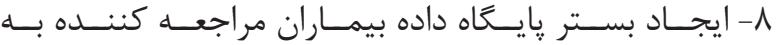

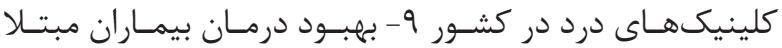

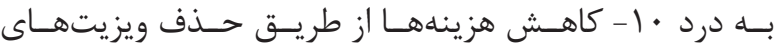

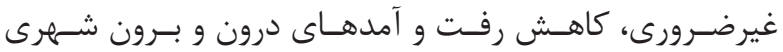

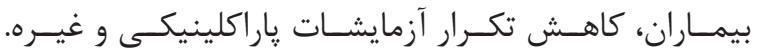

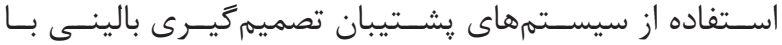

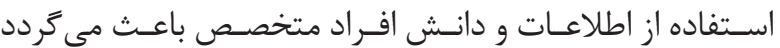

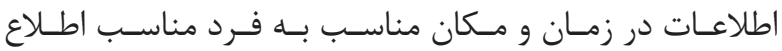

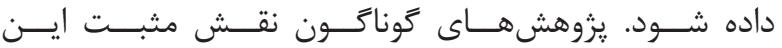

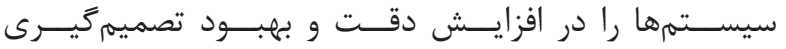

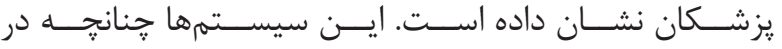

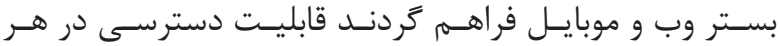

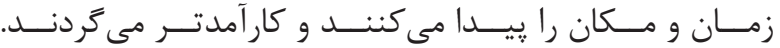

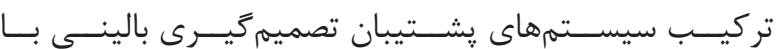

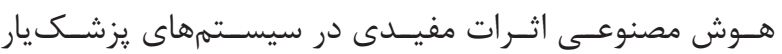

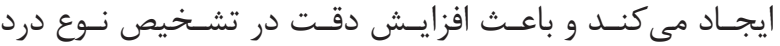

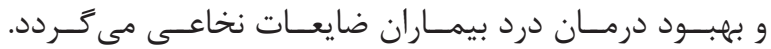

1. Fishman SM, Ballantyne JC, Rathmell JP. Bonica's management of pain. $4^{\text {th }}$ ed. Lippincott Williams \& Wilkins. 2009.

2. Turk DC, Dworkin RH. What should be the core outcomes in chronic pain clinical trials? Arthritis Res Ther. 2004; 6(4): 151-4.

3. Mohammadzadeh F, Faghihzadeh S, Asadi Lari M, Vaez Mahdavi MR, Arab Kheradmand J, Noorbala AA, et al. A fairly comprehensive survey of chronicpain in Iranian population: prevalence, risk factors, and impact on daily life. Health Scope. 2015; 4(3): e25467. doi:

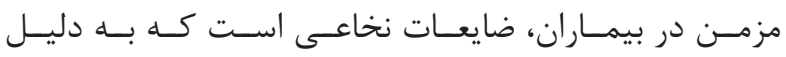

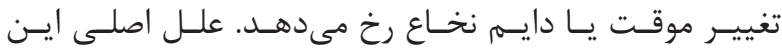

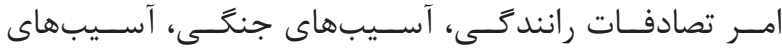

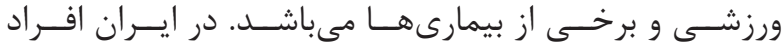

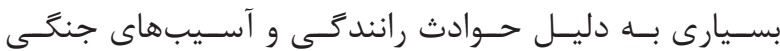

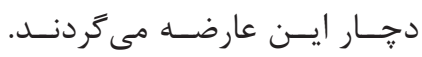

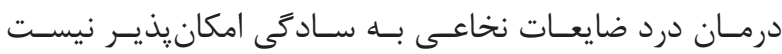

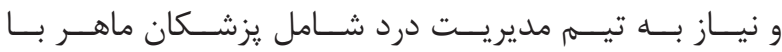

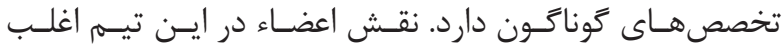

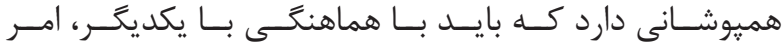

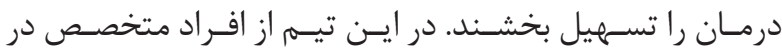

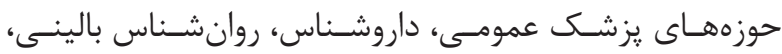

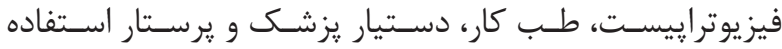

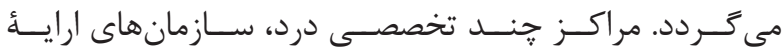

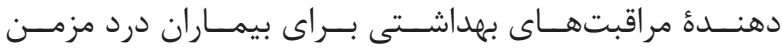

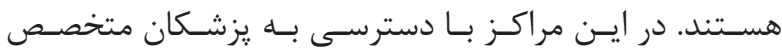

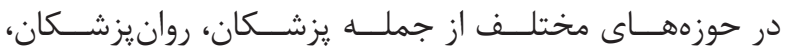

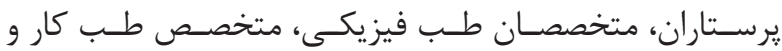

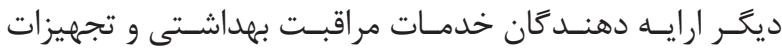

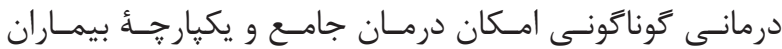

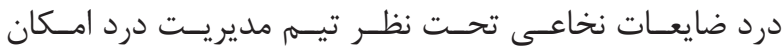

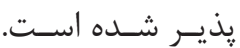

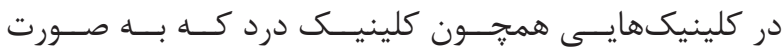

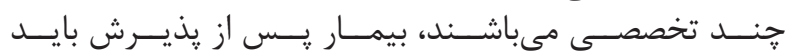

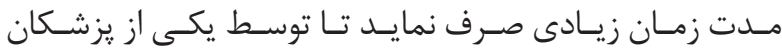

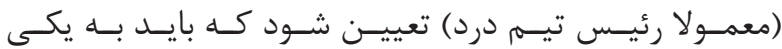

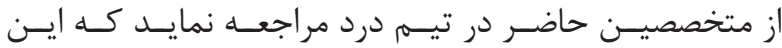

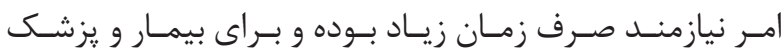

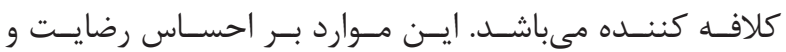

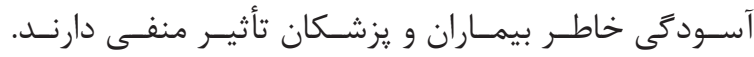

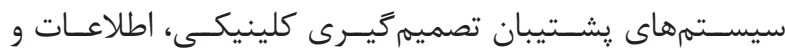

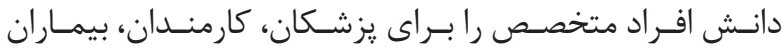

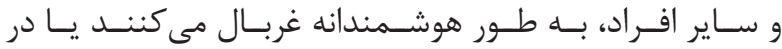

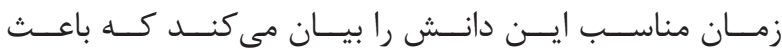

$10.17795 /$ jhealthscope-25467.

منابع

4. Breivik H, Borchgrevink PC, Allen SM, Rosseland LA, Romundstad L, Hals EB, et al. Assessment of pain. Br J Anaesth. 2008; 101(1): 17-24.

5. Pain management. https://en.wikipedia.org/w/index. php?title=Pain_management\&oldid=673213088.

6. Bassols A, Bosch F, Campillo M, Cañellas M, Baños JE. An epidemiological comparison of pain complaints in the general population of Catalonia (Spain). Pain. 1999; 83(1): 9-16. 
7- Sternbach RA. Pain and hassles' in the United States: findings of theNuprin pain report.Pain. 1986;27(1):69-80.

8. Accidents are the most important factor of spinal cord injury in Iran http://www.isaarsci.ir/eduartsci\%20folder/ eduartsci465.htm.

9. Iran ranked 189th amongst 190 countries in road accidents. http://www.migna.ir/vdcauon6.49nwo15kk4. html.

10. Iranian legal medicine organization. http://www. lmo.ir/index.aspx? siteid=1\&pageid=2370.

11. Car accident in Nowruz damage 2000 million Toman to country. http://www.ilna.ir/fa/tiny/news-357698.

12. Ashburn MA, Staats PS. Management of chronic pain. The Lancet. 1999; 353(9167): 1865-9.

13. Carter JJ. Watson AC, Sminkey PV. Pain management: screening and assessment of pain as part of a comprehensive case management process. Prof Case Manag. 2014; 19(3): 126-34.

14. Gatchel RJ, McGeary DD, McGeary CA, Lippe B. Interdisciplinary chronic pain management: past, present, and future. Am Psychol. 2014; 69(2): 119-30.

15. Patil AA, Loden J, Raikar S, Fowler RL. Spinal cord stimulation (SCS) for pain control in a quadriplegic patient: a case report. OJMN. 2016; 6: 25-8.

16. Rose M, Robinson J, Ells P, Cole J. Pain following spinal cord injury: result from a postal survey. Pain. 1988; 34(1): 101-2.

17. Turk DC, Dworkin RH. What should be the core outcomes in chronic pain clinical trials? Arthritis Res Ther. 2004; 6(4): 151-4.

18. Mohammadzadeh F, Faghihzadeh S, Baghestani AR, Asadi Lari M, Vaez Mahdavi M, Arab Kheradmand J, et al. Epidemiology of chronic pain in Tehran small area estimation of its prevalence in Tehran neighborhoods by Bayesian approach (Urban HEART-2 study). Iran J Epidemiol. 2013; 9(1): 19-31.

19. Sleed M, Eccleston C, Beecham J, Knapp M, Jordan A. The economic impact of chronic pain in adolescence: methodological considerations and a preliminary costsof-illness study. Pain. 2005; 119(1-3): 183-90.

20. Chin LS. Spinal cord injuries: practice essentials. http://emedicine.medscape.com/article/793582-overview.
21. Campagnolo DI, Kirshblum S, Nash MS, Heary RF, Gorman PH. Spinal cord medicine. $2^{\text {nd }}$ ed. Lippincott Williams \& Wilkins. 2011.

22. Siddall PJ, Taylor DA, Cousins MJ. Classification of pain following spinal cord injury. Spinal Cord. 1997; 35(2): 69-75.

23.Westgren N, Levi R. Quality of life and traumatic spinal cord injury. Arch Phys Med Rehabil. 1998; 79(11): 1433-9.

24. Hagen EM, Rekand T. Management of neuropathic pain associated with spinal cord injury. Pain Ther. 2015; 4(1): 51-65.

25. Bonica JJ. Pain management: past and current status including role of the anesthesiologist. Stanley TH, Ashburn MA, Fine PG. Anesthesiology and pain management. Springer. 1991; p. 1-30.

26. Fordyce WE, Fowler RS Jr, Lehmann JF, Delateur BJ, Sand PL, Trieschmann RB. Operant conditioning in the treatment of chronic pain. Arch Phys Med Rehabil. 1973; 54(9): 399-408.

27. Turk D, Stacey BR. Multidisciplinary pain centers in the treatment of chronic back pain. Frymoyer JW. Adult spine principles and practice 2VOL. $2^{\text {nd }}$ ed. Lippincottraven Publishers. 1997; p. 253-74.

28. Pain clinic. https://fa.wikipedia.org /w/index.

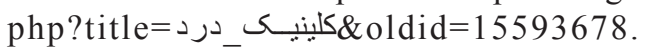

29. Conway J, Higgins I. Literature review: models of care for pain management. Sax Inst Syd. 2011.

30. O'Brien JA. Introduction to information systems: essentials for the internetworked e-business enterprise. 10th ed. Mcgraw-Hill College. 2000.

31. Lippeveld T, Sauerborn R, Bodart C. Design and implementation of health information systems. World Health Organization Geneva. 2000.

32. Wager KA, Lee FW, Glaser JP. Managing health care information systems: a practical approach for health care management. $3^{\text {th }}$ ed. Jossey-Bass. 2013.

33. Wyatt JC, Wyatt SM. When and how to evaluate health information systems? Int J Med Inform. 2003; 69(2-3): 251-9.

34. Asefzadeh S, Fozounkhah S. Challenges in evaluation of the health information systems. J Qazvin Univ Med Sci. 2007; 11(2): 61-70. 
35. Baig MM, Gholamhosseini H. Smart health monitoring systems: an overview of design and modeling. J Med Syst. 2013; 37: 9898. doi: 10.1007/ s 10916-012-9898-z.

36. Tulchinsky TH, Varavikova EA. The new public health: an introduction for the 21 st century. $1^{\text {st }}$ ed. Academic Press. 2000.

37. Shortliffe EH, Cimino JJ. Biomedical informatics: computer applications in health care and biomedicine. $4^{\text {th }}$ ed. Springer Science \& Business Media. 2013.

38. Ansarizadeh T, Yazdanpanah A, Ghavam A, Ghavamabadi I. Effect of hospital information system on performance of medical documents wards for Ahvaz selected hospitals. Int J Innov Knowl Concepts. 2016; 2(3). 43-6.

39. Dargahi H, Ghazi Saeedi M, Safdari R, Hamedan M. A survey of clinical information system process in general hospitals of Tehran university of medical sciences. Payavard Salamat. 2010; 4(2-1): 31-43.

40. Kaplan B, Brennan PF, Dowling AF, Friedman CP, Peel V. Toward an informatics research agenda: key people and organizational issues. J Am Med Inform Assoc. 2001; 8(3): 235-41.

41. Osheroff JA, Teich JM, Middleton B, Steen EB, Wright A, Detmer DE. A road map for national action on clinical decision support. J Am Med Inform Assoc. 2007; 14(2): 141-5.

42. Musen MA, Middleton B, Greenes RA. Clinical decision-support systems. Shortliffe EH, Cimino JJ. Biomedical informatics: computer applications in health care and biomedicine (health informatics). $4^{\text {th }}$ ed. Springer. 2014; p. 643- 674.

43. Osheroff JA, Pifer EA, Sittig DF, Jenders RA, Teich JM. Clinical decision support implementers' workbook. Himss. 2004.

44. Pombo N, Araújo P, Viana J. Knowledge discovery in clinical decision support systems for pain management: a systematic review. Artif Intell Med. 2014; 60(1): 1-11.

45. Ariaei M, Sarafi Nejad A, Kouti J, Mehdipour Z, Bahaadinbeigy K. Role of clinical decision supporting systems in prevention of medical errors from the perspective of health care staff in university hospitals of Kerman university of medical sciences, Iran. Health Inf Manag. 2012; 9(5): 712-23.

46. Khalifa M. Clinical decision support: strategies for success. Procedia Comput Sci. 2014; 37: 422-7.

47. Gerard MN, Trick WE, Das K, Charles-Damte M, Murphy GA, Benson IM. Use of clinical decision support to increase influenza vaccination: multi-year evolution of the system. J Am Med Inform Assoc. 2008; 15(6): 776-9.

48. Teich JM, Osheroff JA, Pifer EA, Sittig DF, Jenders RA. Clinical decision support in electronic prescribing: recommendations and an action plan: report of the joint clinical decision support workgroup. J Am Med Inform Assoc. 2005; 12(4): 365-76.

49. Ohmann C, Moustakis V, Yang Q, Lang K. Evaluation of automatic knowledge acquisition techniques in the diagnosis of acute abdominal pain. Acute abdominal pain study group. Artif Intell Med. 1996; 8(1): 23-36.

50. Roshanov PS, Misra S, Gerstein HC, Garg AX, Sebaldt RJ, Mackay JA, et al. Computerized clinical decision support systems for chronic disease management: a decision-maker-researcher partnership systematic review. Implement Sci IS. 2011; 6: 92. doi: 10.1186/1748-5908-6-92.

51. Neill DB. Using artificial intelligence to improve hospital inpatient care. IEEE Intelligent Systems. 2013; 28(2): $92-5$.

52. Basheer IA, Hajmeer M. Artificial neural networks: fundamentals, computing, design, and application. J Microbiol Methods. 2000; 43(1): 3-31.

53. Foster KR, Koprowski R, Skufca JD. Machine learning, medical diagnosis, and biomedical engineering research commentary. Biomed Eng Online. 2014; 13: 94. doi: 10.1186/1475-925X-13-94.

54. Bayesian network. https://en.wikipedia.org/wiki/ Bayesian_network.

55. Verma SK, Chun S, Liu BJ. A web-based neurological pain classifier tool utilizing Bayesian decision theory for pain classification in spinal cord injury patients. Proc SPIE. 2014; 9039: doi: 10.1117/12.2044434. 